\title{
Forward Error Correction Code for MIMO-OFDM System in AWGN and Rayleigh Fading Channel
}

\author{
R.Krishnamoorthy \\ Department of CSE \\ Anna University-Chennai, BIT Campus \\ Trichy-24
}

\author{
N.S.Pradeep \\ Department of ECE \\ Anna University-Chennai, BIT Campus \\ Trichy-24
}

\begin{abstract}
Orthogonal Frequency Division Multiplexing (OFDM) is a popular method for high data rate wireless transmission. OFDM may be combined with antenna arrays at the transmitter and receiver to increase the diversity gain and/or to enhance the system capacity on time-variant and frequencyselective channels, resulting in a Multiple-Input MultipleOutput (MIMO) configuration. To investigate the performance of MIMO-OFDM system, its physical layer is simulated using MATLAB and Bit Error Rate (BER) performance is observed. Further performance improvement is achieved using Forward Error Correction (FEC) codes. The goal of this paper is to compare different FEC codes in Additive White Gaussian Noise (AWGN) and Rayleigh fading channel and propose a suitable code for MIMO-OFDM system. Three codes, Reed Solomon-Convolution Code (RSCC), Convolutional Turbo Code (CTC) and Low Density Parity Check code (LDPC) are considered for this purpose. It is found that CTC gave a coding gain of $0.2 \mathrm{~dB}$ and $0.25 \mathrm{~dB}$ than its rival LDPC in AWGN and Rayleigh fading channel respectively.
\end{abstract}

\section{General Terms}

Digital Communication, Error Correction Codes, Wireless Broadband Communication.

\section{Keywords}

BER, FEC, RS-CC, CTC, LDPC, MIMO-OFDM.

\section{INTRODUCTION}

Orthogonal frequency division multiplexing (OFDM) has become a popular technique for transmission of signals over wireless channels. OFDM has been adopted in several wireless standards such as digital audio broadcasting (DAB), digital video broadcasting (DVB-T), the IEEE 802.11a [13] local area network (LAN) standard and the IEEE 802.16e [1] metropolitan area network (MAN) standard. OFDM is also being pursued for dedicated short-range communications (DSRC) for road side to vehicle communications and as a potential candidate for fourth-generation (4G) mobile wireless systems, particularly WIMAX [5].

Multiple antennas can be used at the transmitter and receiver, an arrangement called a multiple-input multiple-output (MIMO) system. A MIMO system takes advantage of the spatial diversity that is obtained by spatially separated antennas in a dense multipath scattering environment. MIMO systems may be implemented in a number of different ways to obtain either a diversity gain to combat signal fading or to obtain a capacity gain. Generally, there are three categories of MIMO techniques. The first aims to improve the power efficiency by maximizing spatial diversity. Such techniques include delay diversity, space-time block codes (STBC) [14], [15] and space-time trellis codes (STTC) [16]. The second class uses a layered approach to increase capacity. One popular example of such a system is V-BLAST suggested by Foschini et al. [17] where full spatial diversity is usually not achieved. Finally, the third type exploits the knowledge of channel at the transmitter. It decomposes the channel coefficient matrix using singular value decomposition (SVD) and uses these decomposed unitary matrices as pre- and postfilters at the transmitter and the receiver to achieve near capacity [18].

This paper is focused on the physical layer of OFDM. We have simulated 802.16e PHY and evaluated its performance over different FEC. In MIMO-OFDM, for the purpose of FEC, CC is mandatory, Reed Solomon Code (RS), Convolutional Turbo code (CTC), Block Turbo code and LDPC are optional. Performance is evaluated by considering three coding schemes Reed Solomon-Convolution Code (RSCC), Convolutional Turbo Code (CTC) and Low Density Parity Check code (LDPC).

The paper is organized as follows: System model and OFDM description is given in section 2. A brief overview of FEC is presented in Section 3. Simulation parameters and results are provided in section 4 .

\section{OFDM OVERVIEW}

The IEEE 802.16 standard supports multiple physical specifications due to its modular nature. The 802.16e OFDMA PHY is based on Orthogonal Frequency Division Multiple Access (OFDMA) modulation, which includes OFDM modulation and subcarrier allocation [2], [3].

\subsection{OFDM}

OFDM belongs to a family of transmission schemes called multicarrier modulation, which is based on the idea of dividing a given high-bit-rate data stream into several parallel lower bit-rate streams and modulating each stream on separate carriers, called subcarriers, or tones. OFDM is a spectrally efficient version of multicarrier modulation, where the subcarriers are selected such that they all are orthogonal to one another over the symbol duration.

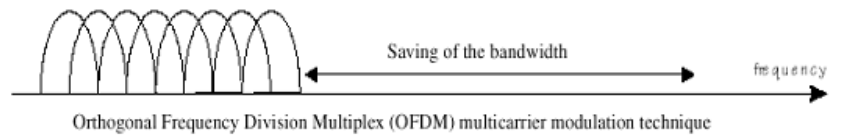

Fig 1: Representation of OFDM 
This avoids the need to have a non-overlapping subcarrier channels to eliminate Inter Carrier Interference (ICI) [4]. Fig.1 shows the advantage of OFDM. An OFDM symbol consists of a number of carriers equal to the size of the Fast Fourier Transform. The OFDM symbols are constructed from data, pilot and null carriers:

- Data carriers - for data transmission.

- Pilot carriers - the magnitude and phase of these carriers are known to the receiver and they are used for channel estimation.

- Null carriers - there are no transmitted energy on these carriers to enable the signal to naturally decay and prevent leakage of energy into adjacent channels.

To support multiple accessing, the data subcarriers are divided into groups that make up sub channels. The subcarriers that make up a sub channel are distributed across all of the available carriers. Particular users are allocated a number of different sub channels to send and receive data [4]. One OFDM symbol can be divided into two parts in time domain: the cyclic prefix (CP) time $\mathrm{T}_{\mathrm{g}}$ and the useful symbol time $\mathrm{T}_{\mathrm{b}}$. The subcarrier orthogonality of an OFDM system can be jeopardized when passed through a multipath channel. CP is used to combat Inter Symbol Interference (ISI) and ICI introduced by the multipath channel. $\mathrm{CP}$ is a copy of the last part of OFDM symbol which is appended to the front of transmitted OFDM symbol. The length of the $\mathrm{CP}\left(\mathrm{T}_{\mathrm{g}}\right)$ or guard time interval must be chosen longer than the maximum delay spread of the target multipath environment [3]. Fig.2 depicts the benefits that arise from $\mathrm{CP}$ addition.

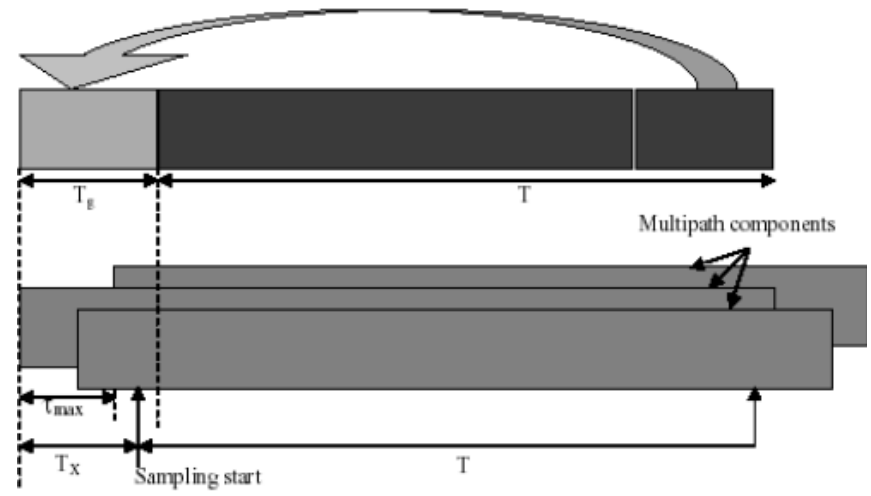

Fig 2: Cyclic Prefix in OFDM

FFT sizes of 128, 512, 1024 or 2048 are defined to be supported by the OFDM specification. Since the sub carrier separation and symbol duration are invariant, the bandwidth of the system could change.

\subsection{Simulation Model}

The simulation for this paper is done on MATLAB. Each block is individually coded. Simulation model is shown in Fig.3. It consists of the following:

1) Source generator: The information bits that will be transmitted are generated using MATLAB function "randint".

2) Modulation: 16-QAM modulation is used in our simulation

3) FEC: RS-CC, CTC and LDPC codes are used.

4) Interleaving: Serial data after FEC block passed through an interleaver block. There are two types of Interleaving, Time Interleaving and Frequency Interleaving. The data symbols are written in the interleaving block in column order, then once the block is full; the symbols are read in row order and transmitted.

5) S/P: Converts serial data into parallel data and vice versa

6) IFFT: An inverse Fourier transform converts the frequency domain data set into samples of the corresponding time domain representing OFDM subcarrier. Specifically IFFT is useful for OFDM because it generates samples of a waveform with frequency component satisfying orthogonality condition.

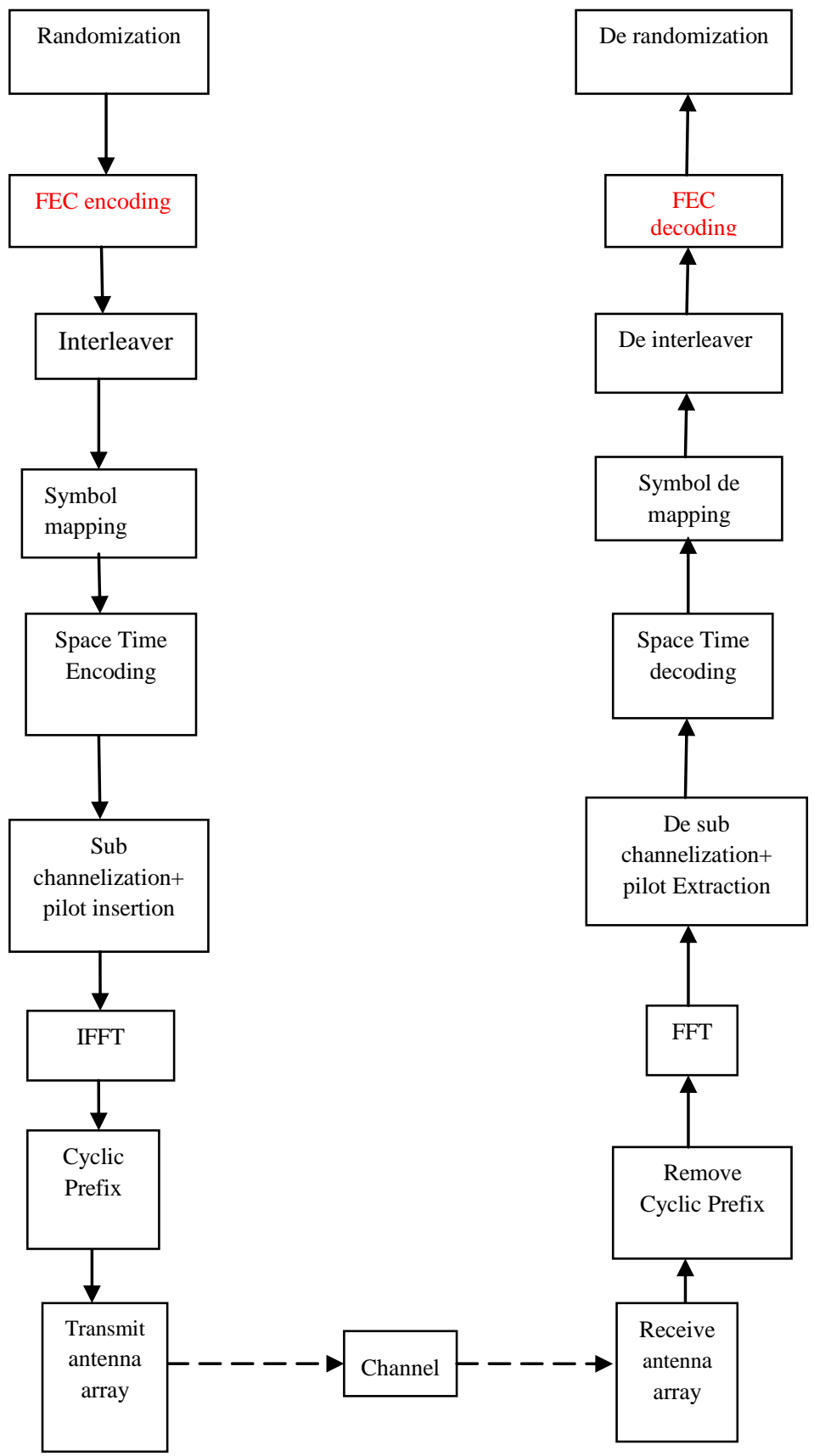

Fig 3: MIMO-OFDM System

7) Cyclic Prefix Addition: In this block numbers of bits falling in $\mathrm{T}_{\mathrm{g}}$ time are added in starting of an OFDM symbol to avoid ISI and ICI.

8) Antenna Array: $2 \times 2$ array is employed with Alamouti Space Time Coding (STC). 


\subsection{AWGN Channel}

In communications, the Additive White Gaussian Noise (AWGN) channel model is one in which the only impairment is the linear addition of wideband or white noise with a constant spectral density (expressed as watts per hertz of bandwidth) and a Gaussian distribution of amplitude. The model does not account for the phenomena of fading, frequency selectivity, interference, nonlinearity or dispersion. However, it produces simple, tractable mathematical models which are useful for gaining insight into the underlying behavior of a system before these other phenomena are considered.

\subsection{Rayleigh Fading Channel}

Rayleigh fading is a reasonable model when there are many objects in the environment that scatter the radio signal before it arrives at the receiver. The central limit theorem holds that, if there is sufficiently much scatter, the channel impulse response will be well-modeled as a Gaussian process irrespective of the distribution of the individual components. If there is no dominant component to the scatter, then such a process will have zero mean and phase evenly distributed between 0 and $2 \pi$ radians. The envelope of the channel response will therefore be Rayleigh distributed. This random variable $R$ will have a probability density function:

$$
p_{R}(r)=\frac{2 r}{\Omega} e^{\frac{-r^{2}}{\Omega}}, r \geq 0
$$

Where $\Omega=E\left(R^{2}\right)$.Often, the gain and phase elements of a channel's distortion are conveniently represented as a complex number. In this case, Rayleigh fading is exhibited by the assumption that the real and imaginary parts of the response are modeled by independent and identically distributed zero mean Gaussian processes so that the amplitude of the response is the sum of two such processes.

\section{FORWARD ERROR CORRECTION (FEC)}

\subsection{Reed Solomon-Convolutional Code (RS-CC)}

In the MIMO-OFDM part, the RS-CC is the only mandatory coding scheme. RS error correction is a coding scheme which works by first constructing a polynomial from the data symbols to be transmitted, and then sending an oversampled version of the polynomial instead of the original symbols themselves [8]. An RS-code is specified as $R S(n, k, t)$ with $l$ bit symbols. This means that the encoder takes $k$ data symbols of $l$ bits each and adds $2 t$ parity symbols to construct an $n$ symbol codeword. Therefore: $\mathrm{n}$ is the number of bytes after encoding; $k$ is the number of data bytes before encoding, and $t$ is the number of data bytes that can be corrected. Then RScode can correct up to $t$ symbols, where $t$ can be expressed as $t=(n-k) / 2$.

After the RS encoding process, data bits are further encoded by a binary CC, which has a native rate of $1 / 2$ and a constraint length of 7 [11]. The generator polynomials used to derive its two output code bits, denoted $\mathrm{X}$ and $\mathrm{Y}$, are specified in the following expressions: $\mathrm{G} 1=171$ OCT for X, and G2 = 133 OCT for Y. Its computations depend not only on the current set of input symbols but on some of the previous input symbols. A trellis description is used for convolution encoding which gives relation how each possible input to the encoder influences the output in shift register. It uses the Viterbi algorithm for decoding [10].

\subsection{Convolutional Turbo Codes (CTC)}

The CTC encoder, including its constituent encoder, uses a double binary Circular Recursive Systematic Convolutional code. The bits of the data to be encoded are alternately fed to encoder A and B, starting with the MSB of the first byte being fed to A [9]. The encoder is fed by blocks of $k$ bits or $N$ couples ( $k=2 * N$ bits). For all the frame sizes, $k$ is a multiple of 8 and $N$ is a multiple of 4 . Further, $N$ shall be limited to: 8 $\leq N / 4 \leq 1024$. The decoding algorithm of classical Turbo Code is still used in CTC [12].

\subsection{Low Density Parity Check Codes (LDPC)}

Low-density parity-check (LDPC) codes are a class of linear block codes. The name comes from the characteristic of their parity-check matrix $\mathrm{H}$ which contains only a few 1's in comparison to the amount of 0's [6].Their main advantage is that LDPC is the first code to allow data transmission rate performance which is very close to the theoretical capacity maximum, the Shannon Limit and linear time complex algorithms for decoding. It is the sparseness of $\mathrm{H}$ which guarantees both a decoding complexity, which increases only linearly with the code length and a minimum distance, which also increases linearly with the code length. With the optional irregular LDPC codes, $\mathrm{k}$ systematic information bits are encoded to $n$ code bits by adding $r=n-k$ parity check bits. An ( $n, j, k)$ LDPC code is specified by a parity check matrix $H$, having $n-k$ rows, $n$ columns and $j 1$ 's per column [7]. In this paper $\mathrm{j}=3$ i.e., all the parity check matrices will have 3 ones per column. We use $1 / 2$ rate encoder and tried to avoid cycle of length-four, where pair of column, both have 1's in particular rows. For decoding a simplified log domain belief propagation decoder using sum-product algorithm is used.

\section{SIMULATION PARAMETERS AND RESULTS}

The MIMO-OFDM parameters used for the simulation are listed below:

No. of transmit antennas: 2

No. of receive antennas: 2

Diversity Technique: Alamouti STC

Primitive Parameters

Carrier frequency $: 2.5 \mathrm{GHz}$

Channel Bandwidth (BW): $5 \mathrm{MHz}$

FFT size $\left(\mathrm{N}_{\mathrm{FFT}}\right): 512$

Cyclic Prefix (CP): $1 / 8$

Oversampling rate (n): 28/25

Channel: AWGN and Rayleigh Fading

Derived Parameters

Sampling frequency $\left(\mathrm{F}_{\mathrm{s}}=\mathrm{nxBW}\right): 5.6 \mathrm{MHz}$

Subcarrier spacing $\left(\Delta \mathrm{f}=\mathrm{F}_{\mathrm{s}} / \mathrm{N}_{\mathrm{FFT}}\right): 10.94 \mathrm{KHz}$

Useful symbol time $\left(\mathrm{T}_{\mathrm{b}}=1 / \Delta \mathrm{f}\right): 91.4 \mu \mathrm{s}$

Cyclic Prefix time $\left(\mathrm{T}_{\mathrm{g}}=\mathrm{CPxT}_{\mathrm{b}}\right): 11.4 \mu \mathrm{s}$

OFDM symbol duration $\left(\mathrm{T}_{\mathrm{s}}=\mathrm{T}_{\mathrm{b}}+\mathrm{T}_{\mathrm{g}}\right): 102.8 \mu \mathrm{s}$

The nominal bandwidth $\mathrm{BW}$ is assumed to be $5 \mathrm{MHz}$. Applying a sampling factor of $n=28 / 25$, yields a sampling frequency $\mathrm{F}_{\mathrm{s}}=5.6 \mathrm{MHz}$. Denoting the useful symbol time by $T_{b}$ and the length of the cyclic prefix by $T_{g}$, the fraction of $G$ $=T_{\mathrm{g}} / \mathrm{T}_{\mathrm{b}}$ was assumed to be $\mathrm{G}=1 / 8$. Carrier frequency considered is $2.5 \mathrm{GHz}$ and is used for NLoS operation.

In this paper physical layer simulation of MIMO-OFDM system is done under Additive White Gaussian Noise (AWGN) and Rayleigh fading channel condition. AWGN is due to the thermal noise predominant in communication receivers. Rayleigh fading is due to the multipath effect. The mobility of the node considered for simulation is $3 \mathrm{~km} / \mathrm{hr}$ to 
support pedestrian speeds. RS-CC, CTC and LDPC FECs are used for simulation and their comparison is made in terms of Bit Error Rate. Reed Solomon is used as outer code and is concatenated with inner convolutional code, which is the mandatory channel coding scheme for MIMO-OFDM. In this paper additional channel coding schemes like CTC and LDPC are also considered which provides better BER performance than RS-CC. Also Least Square (LS) channel estimation is considered. In this simulation, $2 \times 2$ Alamouti Space Time Coding (STC) is taken into account to combat the effect of fading in the system.

The Fig.4 presents the $\mathrm{E}_{\mathrm{b}} / \mathrm{N}_{0}$ versus BER performance of MIMO-OFDM system without FEC (uncoded) and with three FEC codes with 16QAM modulation. The simulations are carried in AWGN channel.

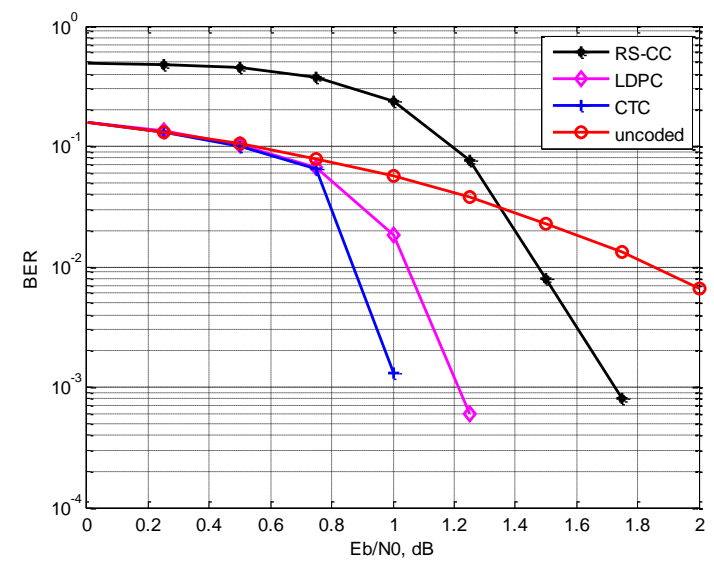

Fig 4: Comparison of different FEC codes for MIMOOFDM in AWGN channel

It is observed from Fig.4 that RS-CC code shows poor result for lower $\mathrm{E}_{\mathrm{b}} / \mathrm{N}_{0}$ values performing inferior to uncoded BER. But for higher values of $\mathrm{E}_{\mathrm{b}} / \mathrm{N}_{0}$, RS-CC gives $0.5 \mathrm{~dB}$ performance improvement than uncoded. LDPC gives further improvement of $0.4 \mathrm{~dB}$ than RS-CC at BER of $8 \times 10^{-4}$ over the curve. On comparing LDPC with CTC, it is observed from Fig.4 that CTC shows improvement in performance of about $0.2 \mathrm{~dB}$ than LDPC at BER of $10^{-3}$ over the curve. Thus it is evident that the use of FEC improved the performance of MIMO-OFDM. CTC is found to be the suitable FEC code for MIMO-OFDM applications, since it provides the lowest BER at lowest $\mathrm{E}_{\mathrm{b}} / \mathrm{N}_{0}$.

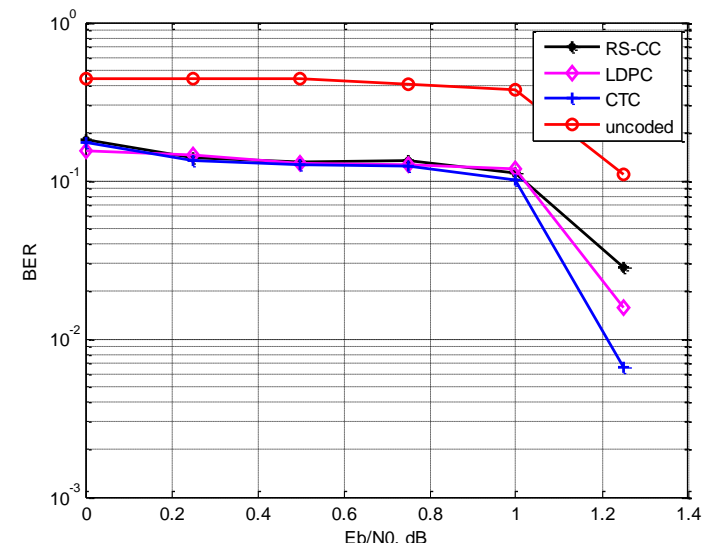

Fig 5: Comparison of different FEC codes for MIMOOFDM in Rayleigh fading channel without STC
Fig.5 depicts the BER performance of MIMO-OFDM for various FEC codes in Rayleigh fading channel without STC. Compared to uncoded, use of FEC improved the BER performance. It is pragmatic that LDPC performs about 0.1 $\mathrm{dB}$ better than the RS-CC at a BER of $2 \times 10^{-2}$, and CTC giving a further improvement of about $0.1 \mathrm{~dB}$ than LDPC. It is also observed that at low $\mathrm{E}_{\mathrm{b}} / \mathrm{N}_{0}(<1 \mathrm{~dB})$ no better performance is achieved for all the FEC schemes. When $\mathrm{E}_{\mathrm{b}} / \mathrm{N}_{0}$ is greater than $1 \mathrm{~dB}$, BER reduces. So in both the channel conditions, CTC is found to give the best performance with reduced BER and is highly robust than LDPC and RS-CC.

Reported in Fig.6 are typical processing times taken for each FEC codes in minutes. From this plot it is clear that the time complexity of CTC is three times higher than RS-CC and LDPC. This is because of the decoding complexity of CTC, which requires large number of iterations to get optimum performance. LDPC and RS-CC required almost same time for processing and they are less complex than CTC.

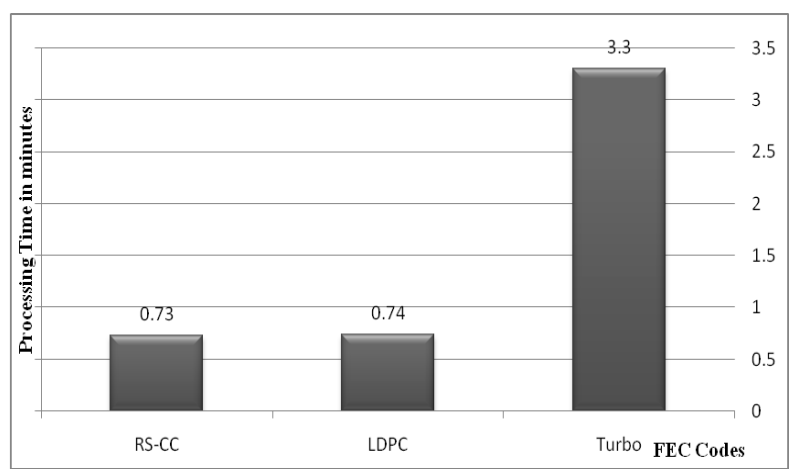

Fig 6: Processing time of each FEC codes

On comparing Fig.4, Fig.5 and Fig.6, it is found that CTC gives the best BER performance but is highly complex than LDPC and RS-CC. So, if reducing BER and robustness is the main design criterion then CTC is the ideal choice. Else if the design consideration is reducing the complexity and requires nominal BER, then LDPC is the suitable choice. Since our goal is to reduce the BER and to provide robustness, CTC is considered for further analysis. Since in Rayleigh fading channel, the performance improvement on using FEC codes is very poor and is unsuitable for MIMO-OFDM applications. So, to combat the effect of fading, Space Time Coding (STC) has been introduced [19] to improve the BER performance of FEC codes in fading channel and is shown in Fig.7.

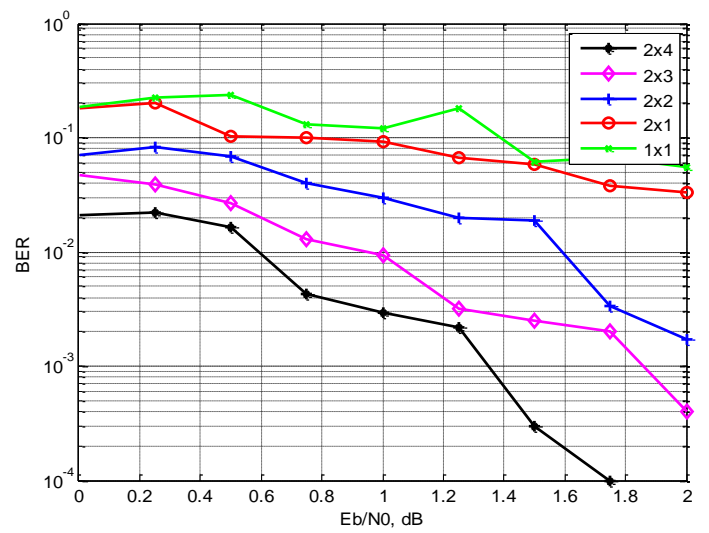

Fig 7: Comparison of STCs for MIMO-OFDM in Rayleigh fading channel 
It is evident from Fig.7 that rather than using single transmit and receive antennas, use of multiple antennas improved the BER performance in fading channel. Since Alamouti STC [14] is used, the number of transmitting antennas is limited to two and the number of receiving antennas is increased from one to four. As the number of receiving antennas are increased, the reliability increases and hence the BER reduces.

With $2 \times 2$ diversity, the performance of fading channel is almost identical to that obtained in AWGN channel. Hence for further analysis involving fading channel, the performance of MIMO-OFDM is improved using $2 \times 2$ STC.

Fig. 8 depicts the comparison of different FEC codes in Rayleigh fading channel with $2 \times 2$ STC. It is observed from figure that RS-CC code shows poor result for lower $\mathrm{E}_{\mathrm{b}} / \mathrm{N}_{0}$ values performing inferior to uncoded BER. But for higher values of $\mathrm{E}_{\mathrm{b}} / \mathrm{N}_{0}$, RS-CC gives $0.2 \mathrm{~dB}$ performance improvement than uncoded. LDPC gives further improvement of $0.3 \mathrm{~dB}$ than RS-CC at BER of $2 \times 10^{-3}$ over the curve. On comparing LDPC with CTC, it is found that later shows improvement in performance of about $0.25 \mathrm{~dB}$ than LDPC at BER of $9 \times 10^{-3}$ over the curve. Thus it is evident that the use of FEC with STC improved the performance of OFDM system by combating the effect of fading. CTC is found to be the suitable FEC code for MIMO-OFDM applications in both AWGN and Rayleigh fading channels.

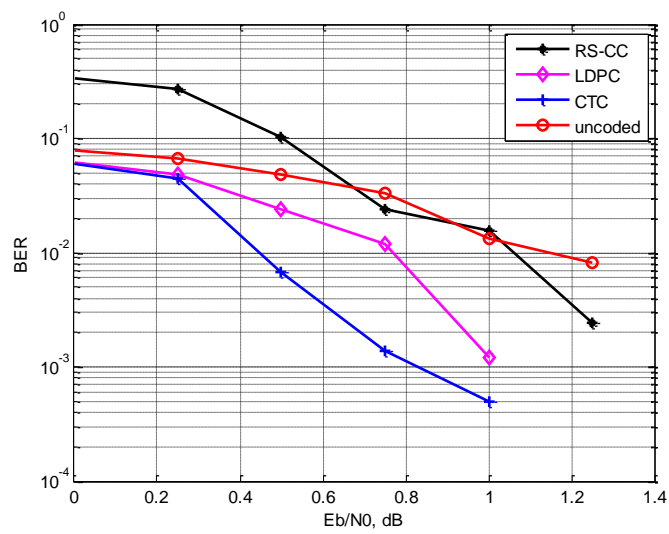

Fig 8: Comparison of different FEC codes for MIMOOFDM in Rayleigh fading channel with $2 \times 2$ STC

\section{CONCLUSION}

The performance of MIMO-OFDM physical layer is improved by using FEC codes. In AWGN channel, RS-CC code shows poor result for lower $\mathrm{E}_{\mathrm{b}} / \mathrm{N}_{0}$ values performing inferior to uncoded BER. But for higher values of $E_{b} / N_{0}$, RS$\mathrm{CC}$ gives $0.5 \mathrm{~dB}$ performance improvement than uncoded. LDPC gives further improvement of $0.4 \mathrm{~dB}$ than RS-CC at BER of $10^{-4}$ over the curve. On comparing LDPC with CTC, it is observed that CTC shows improvement in performance of about $0.2 \mathrm{~dB}$ than LDPC at BER of $10^{-3}$ over the curve. In Rayleigh fading channel without STC, compared to uncoded, use of FEC improved the BER performance. It is observed that FEC with LDPC performs about $0.1 \mathrm{~dB}$ better than RS$\mathrm{CC}$ at a BER of $2 \times 10^{-2}$ over the curve and CTC giving a further improvement of about $0.1 \mathrm{~dB}$ than LDPC. Also the time complexity of CTC is three times higher than LDPC and RS-CC. So, if reducing BER and robustness is the main design criterion then CTC is the ideal choice. Else if the design consideration is reducing the complexity and requires nominal BER, then LDPC is the suitable choice. In Rayleigh fading channel with $2 \times 2$ STC, CTC shows improvement in performance of about $0.25 \mathrm{~dB}$ than LDPC at BER of $10^{-3}$ over the curve.

\section{REFERENCES}

[1] IEEE Standard 802.16-2004, "Standard for Local and Metropolitan area networks", Part 16: Air Interface for Fixed Broadband Wireless Access Systems, October 2004.

[2] IEEE 802.16e/D7, "Draft IEEE Standard for Local and metropolitan area networks, Part 16: Air Interface for Fixed Broadband Wireless Access Systems, Amendment for Physical and Medium Access Control Layers for Combined Fixed and Mobile Operation in Licensed Bands", April 2005

[3] Richard van Nee, Ramjee Prasad "OFDM for Wireless Multimedia Communication”, Artech House Publishers, 2000.

[4] L. J. Cimini Jr., "Analysis and simulation of a digital mobile channel using orthogonal frequency division multiplexing," IEEE Trans. Commun., vol. COM-33, pp. 665-675, July 1985.

[5] Wen-an Zhou; Bing Xie; Jun-de Song, "Link-level Simulation and Performance Estimation of WiMAX IEEE802.16e", 2nd International Conference on Pervasive Computing and Applications, Page(s):667 671, July 2007

[6] R.G. Gallager, "Low density parity check codes," IRE Trans. Inform. Theory, vol. IT-8, no. 1, pp. 21-28, Jan. 1962.

[7] J. Hou, P.H. Siegel, and L.B. Milstein, "Performance analysis and code optimization of low density paritycheck codes on Rayleigh fading channels," IEEE J. Select. Areas Commun., vol. 19, no. 5, pp. 924-934, May 2001.

[8] W.J. Gross, F.R. Kschischang, R. Kotter, and P.G. Gulak, "Applications of algebraic soft-decision decoding of Reed-Solomon codes," IEEE Trans. Commun., vol. 54, no. 7, pp. 1224-1234, Jul. 2006.

[9] C. Anghel, A. A. Enescu, O. Bugiugan, and R. Cacoveanu "FPGA implementation of a CTC Decoder for H-ARQ compliant WiMAX systems," Proc. International Conference on Design \& Technology of Integrated Systems, Morocco, pp. 82-86, 2007.

[10] Hagenauer, J. Hoeher, German Aerosp. Res. Establ., “A Viterbi Algorithm with Soft-Decision Outputs and its Applications", Global Publication, vol.3 pp. 1680-1686, 1989.

[11] S. Benedetto and G. Montorsi, "Design of parallel concatenated convolutional codes," IEEE Trans. Commun., vol. 44, no. 5, pp. 591-600, May 1996.

[12] Jonson P.Woodard, Lajos Hanzo, "Comparative Study of Turbo Decoding Techniques: An Overview", IEEE Transactions on vehicular technology, vol.49, no.6, pp. 2208-2233, November 2000.

[13] Part 11: Wireless LAN Medium Access Control (MAC) and Physical Layer (PHY) Specifications: High-Speed Physical Layer in the $5 \mathrm{GHz}$ Band, IEEE Standard 802.11a-1999. 
[14] S. Alamouti, "A simple transmit diversity technique for wireless communications," IEEE J. Select. Areas Commun., vol. 16, pp. 1451-1458, Oct. 1998.

[15] V. Tarokh, H. Jafarkhani, and A. R. Calderbank, "Spacetime block codes from orthogonal designs," IEEE Trans. Inform. Theory, vol. 45, pp. 1456-1467, July 1999.

[16] V. Tarokh, N. Seshadri, and A. R. Calderbank, "Spacetime codes for high data rate wireless communication: Performance criterion and code construction," IEEE Trans. Inform. Theory, vol. 44, pp. 744-765, Mar. 1998.
[17] P. W. Wolniansky, G. J. Foschini, G. D. Golden, and R. A. Valenzuela, "V-Blast: An architecture for realizing very high data rates over the rich-scattering channel," in Proc. Int. Symp. Signals, Systems and Electronics (ISSE 1998), pp. 295-300.

[18] J. Ha, A. N. Mody, J. H. Sung, J. Barry, S. Mclaughlin, and G. L. Stuber, "LDPC coded OFDM with Alamouti/SVD diversity technique, "J. Wireless Pers. Commun., vol. 23, no. 1, pp. 183-194, Oct. 2002.

[19] Sezginer, S. Sari, H. and Biglieri, E. "A Comparison of Full-Rate Full- Diversity $2 \times 2$ Space-Time Codes for WIMAX Systems", Proc. Spread Spectrum Techniques and Applications, Bologna, Italy, pp. 91-96, 2008. 